\title{
INFLUÊNCIA DO TEMPO DO PROCESSO DE CURTIMENTO NA QUALIDADE DOS COUROS DE COELHOS (O. CUNNICULUS)
}

\author{
INFLUENCE OF TIME OF THE TANNING PROCESS ON THE QUALITY OF \\ THE RABBIT'S SKIN (ORYCTOLAGUS CUNNICULUS)
}

\author{
Franco, M.L.R.S. ${ }^{\text {* }}$, Prado, M. ${ }^{1}$, Domingues, M.C. ${ }^{1}$, Gasparino, E. ${ }^{1}$, Scapinello, C. ${ }^{1}$ \\ e Silva, S.C.C. ${ }^{1}$
}

1Universidade Estadual de Maringá. Maringá-Paraná. Brasil. *mlrsouza@uem.br

\section{PaLAVRAS ChaVE ADICIONAIS}

Pele de coelho. Resistência do couro. Testes físico-mecânicos.

\section{RESUMO}

O experimento foi conduzido para avaliar a qualidade dos couros de coelhos em diferentes partes da superfície e orientações, submetidos a dois diferentes tempos de processamento. Foram utilizadas 40 peles de coelhos, da raça Nova Zelândia Branco, abatidos aos 70 dias de idade. Foi utilizado um delineamento inteiramente casualizado em esquema de parcelas subdivididas, tendo nas parcelas os tratamentos ( $T 1=$ tempo normal; $\mathrm{T} 2=$ tempo contínuo) e nas subparcelas as posições de corte no couro ( $\mathrm{P} 1=$ anterior longitudinal; $\mathrm{P} 2$ = anterior transversal; $\mathrm{P} 3=$ posterior longitudinal e P4= posterior transversal), com dez repetições por tratamento. As etapas aplicadas no processo de curtimento foram: remolho, descarne, caleiro ( $3 \%$ de sulfeto de sódio e $4 \%$ de cal), desencalagem, purga, desengraxe, píquel, curtimento $(6 \%$ de sais de cromo), neutralização, recurtimento ( $4 \%$ sais de cromo), tingimento, engraxe (6\% de óleos sulfatados), secagem e amaciamento. A espessura dos couros foi maior $(p<0,05)$ quando se utilizou a técnica de curtimento com tempo contínuo $(1,03$ $\mathrm{mm})$. O tempo de curtimento proporcionou diferentes valores de tração em todas as posições de retirada do corpo de prova, exceto para anterior transversal. A região posterior do couro apresentou menor tração independente do tempo de curtimento (normal= $6,01 \mathrm{~N} / \mathrm{mm}^{2}$ e contínuo= $3,54 \mathrm{~N} / \mathrm{mm}^{2}$ ). A região anterior independente da posição (longitudinal $=37,28 \mathrm{~N} / \mathrm{mm}$ ou transversal= $35,71 \mathrm{~N} / \mathrm{mm}$ ), apresentou maior resistência

Recibido: 6-4-11. Aceptado: 23-2-12.

\section{Additional KeYWORDS}

Physico-mechanical tests. Rabbit skin. Resistance of leather.

ao rasgamento comparado a região posterior (longitudinal= 26,78 N/mm e transversal= 25,94 N/ $\mathrm{mm}$ ) nos couros curtidos com tempo normal. Curtimento em tempo normal proporcionou menor elasticidade aos couros.

Os couros devem ser curtidos pelo método com tempo normal de processamento, por proporcionarem maior resistência a tração e rasgamento superiores em relaçao aos curtidos em tempo contínuo. A região anterior apresentou maior resistência comparada a região posterior para rasgamento progressivo, independente do tempo de curtimento utilizado. Os couros na região posterior longitudinal apresentaram menor resistência a tração, independente do tempo de processamento aplicado. Deve-se ressaltar que o curtimento em período normal proporcionou couros com menor elasticidade, mas pelo teste de rasgamento progressivo e alongamento, os valores estão dentro dos padrões exigidos para confecção de vestuários seguindo os valores BASF (2005).

\section{SUMMARY}

This experiment was carried out to evaluate the quality of the rabbit skins in different parts and orientations of the surface, submitted at different time of processing. The rabbits were slaughtered with 70 days of age and forty skins were used. It was used a completely randomized design in a 2 $x 4$ factorial treatment, being in two times of 
processing $(\mathrm{T} 1=$ regular time and $\mathrm{T} 2=$ continuous time) and four positions of cut in the skin ( $P 1=$ fore longitudinal and $\mathrm{P} 2=$ fore transversal; $\mathrm{P} 3=$ hind longitudinal and $\mathrm{P} 4=$ hind transversal). The steps of the tanning process were soaking, fleshing, liming ( $3 \%$ of sodium sulphide and $4 \%$ of lime), alkaline products remotion, purge, degrease, pickling, tanning ( $6 \%$ of chrome salts), neutralization, retanning ( $4 \%$ of chrome salts), dying, grease ( $6 \%$ of sulfacant oils), dryness and softening. The thickness of the rabbit skins was significantly higher $(p<0.05)$ when the continuous time technique was used $(1.03 \mathrm{~mm})$. The time of tanning gave different values of traction in all positions of the samples, except for the fore transversal. The hind region regardless of the time showed a less resistant skin to traction (regular time $=6.01 \mathrm{~N} / \mathrm{mm}^{2}$ and continuous time $=3.54 \mathrm{~N} /$ $\mathrm{mm}^{2}$ ). The fore region regardless if it is a longitudinal way $(37.28 \mathrm{~N} / \mathrm{mm})$ or transversal $(35.71 \mathrm{~N} / \mathrm{mm})$, showed a higher resistance to tearing compared to the hind region (longitudinal $=26.78 \mathrm{~N} / \mathrm{mm}$ and transversal $=25.94 \mathrm{~N} / \mathrm{mm}$ ) in regular time. The technique used in the regular time tanning offered a less elasticity to the rabbit skin in relation to the continuous time. The skins must be tanning by regular time because offer more resistance to traction and superior tearing than skins tanned by continuous time. The fore region showed more resistance than hind region for progressive tearing in all times of tanning. The skins of the hind longitudinal region regardless of the time showed a less resistant to traction. The regular time of tanning gave skins with less elasticity, but for progressive tearing and stretching test, the values are in the pattern for be used in clothing manufacturing, according to BASF (2005).

\section{INTRODUÇÃO}

A cunicultura, como atividade de exploração animal intensiva, tem utilizado, cada vez mais, técnicas que melhorem a produtividade, destacando-se aquelas relacionadas à reprodução (Fernández-Carmona et al., 2000). Os mesmos autores relatam que há grandes perspectivas de crescimento e demanda no consumo mundial de carne de coelho.

Nos últimos 40 anos a produção de coelhos evoluiu de um sistema de exploração familiar, para uma atividade econômica industrial, similar àquelas desenvolvidas para outras espécies animais, como aves e suínos. Esse fenômeno tem sido favorecido por circunstâncias econômicas, de mercado e pelas características produtivas da espécie. No entanto, a industrialização tem sido mais intensa nos países europeus, onde o consumo da carne de coelho faz parte do hábito alimentar da população (Carabaño, 2000).

No Brasil, a criação de coelhos está voltada para produção da carne. No entanto, grande parte dos subprodutos do abate, particularmente a pele, o cérebro e o sangue ainda são pouco utilizados. O processamento e industrialização destes subprodutos podem contribuir de forma decisiva para a viabilização da cunicultura (Scapinello, 1986). A pele, após processamento, pode ser utilizada nas confecções e indústrias de artefatos de couro ou então para produção de gelatina; os pêlos para fabricação de feltro e as patas dianteiras e cauda na confecção de chaveiro.

A pele do coelho é um subproduto que pode ser beneficiado, tanto para uso na peleteria (com pêlos), como transformada em couro (sem a presença de pêlos). Após o processo de curtimento, esse couro pode ser considerado uma matéria-prima de elevada qualidade e beleza pela sua suavidade ao toque e com um desenho de flor (superfície da pele sem pêlo) extremamente delicado. Atualmente, essas peles estão sendo, descartadas ou subutilizadas, pela falta de adequadas técnicas de curtimento, sistema de conservação e armazenamento.

Para oferecer ao mercado um produto com qualidade e com um preço competitivo, a primeira medida a ser tomada pelos curtidores é a maximização dos processos de curtimento, com redução dos custos.

Normalmente, um processo de curtimento leva em torno de 52 horas para peles delicadas e de animais de pequeno porte, incluído os tempos em que as peles permanecem em repouso (píquel e curti-mento e após a basificação). A rapidez no processamento 


\section{TEMPOS DAS ETAPAS NO CURTIMENTO}

permitirá a otimização dos equipamentos dentro do curtume.

Dessa forma, este trabalho teve como objetivo avaliar a qualidade dos couros de coelhos na região anterior e posterior e nas duas posições (longitudinal e transversal), submetidos a diferentes tempos de processamento.

\section{MATERIALE MÉTODOS}

O experimento foi conduzido no Laboratório de processamento de peles de peixes e demais espécies de pequeno e médio porte, do Departamento de Zootecnia da Universidade Estadual de Maringá.

Foram utilizadas 40 peles de coelhos (Oryctolagus cuniculus), da raça Nova Zelândia Branco, abatidos aos 70 dias de idade, distribuídas em um delineamento inteiramente casualizado em esquema de parcelas subdivididas, tendo nas parcelas os tratamentos $(\mathrm{T}=$ tempo normal, $49 \mathrm{~h}, \mathrm{~T} 2=$ tempo contínuo, $10 \mathrm{~h}$ e $10 \mathrm{~min}$.) e nas subparcelas as posições de corte no couro $(\mathrm{P} 1=$ anterior longitudinal, $\mathrm{P} 2=$ anterior transversal, $\mathrm{P} 3=$ posterior longitudinal e $\mathrm{P} 4=$ posterior transversal), com dez repetições.
Por ocasião do abate dos animais, as peles foram embaladas em sacos plásticos e armazenadas em freezer a $-18^{\circ} \mathrm{C}$ até o momento do processamento.

O curtimento das peles foi realizado de acordo com as etapas de processamento descritas por Hoinacki (1989) e Souza (2004), com algumas modificações. As etapas foram o remolho, descarne, caleiro (3\% de sulfeto de sódio e 4\% de cal), desencalagem, purga, desengraxe, píquel, curtimento ( $6 \%$ de sais de cromo), neutralização, recurtimento ( $4 \%$ sais de cromo), tingimento, engraxe ( $6 \%$ de óleos sulfatados), secagem e amaciamento.

As peles foram descongeladas, remolhadas, descarnadas e pesados dois lotes para submeter aos curtimentos com tempos diferentes de processamento. O cálculo dos tempos utilizados para processar cada lote de pele, está apresentado na tabela I. O lote um foi processado de forma padrão segundo Souza (2004) com intervalos recomendados entre as etapas de curtimento e após basificação, totalizando $49 \mathrm{~h}$, enquanto o lote dois a forma contínua, em $10 \mathrm{~h} 10 \mathrm{~min}$ (sem tempo de repouso entre etapas e com algumas alterações no tempo do processo,

Tabela I. Etapas do processo de curtimento com os tempos utilizados para as peles de coelhos. (Steps of tanning process of two methods used to rabbit skins).

\begin{tabular}{ll}
\hline Processo de curtimento normal & Processo de curtimento contínuo \\
\hline Caleiro - Fulão por 2 h e 30 min & Caleiro - Fulão por 2 h 30 min \\
Desencalagem - Fulão por 1 h e 30 min & Desencalagem - Fulão por 60 min \\
Purga - Fulão por 60 min & Purga - Fulão por 30 min \\
Desengraxe - Fulão por 20 min & Desengraxe - Fulão por 20 min \\
Píquel - Fulão por 40 min & Píquel - Fulão por 40 min \\
Curtimento - Fulão parado por $13 \mathrm{~h}$ & Curtimento - Fulão por 60 min \\
Basificação - 4 h & Basificação - 60 min \\
Repouso - 19 h e 30 min & Neutralização - Fulão por 40 min \\
Neutralização - Fulão parado por 60 min & Recurtimento - Fulão por 60 min \\
Recurtimento e tingimento - Fulão por $4 \mathrm{~h}$ & Engraxe e tingimento - Fulão por 60 min \\
Engraxe - Fulão por 60 min & Fixação - Fulão por 30 min \\
Fixação - Fulão por 30 min & \\
Tempo total - 49 h & \\
\hline
\end{tabular}


passando para o tempo mínimo a ser realizado na etapa).

Após o curtimento das peles, foram retirados os corpos de prova para os testes de determinação da resistência à tração e alongamento (ABNT, 1997) e a determinação da força de rasgamento progressivo (ABNT, 2005a). Os corpos de prova foram obtidos das regiões anterior e posterior e no sentido longitudinal e transversal em relação ao eixo cefalo-caudal (figura 1), com auxílio de um balancim e, em seguida, foram levados para um ambiente climatizado em torno de $23 \pm$ $2{ }^{\circ} \mathrm{C}$ e umidade relativa do ar de $50 \pm 5 \%$, por 48 horas (ABNT, 2006). Foram determinadas as medidas de espessura de cada amostra (ABNT, 2005b). Para os testes de resistência foi utilizado o dinamômetro da marca EMIC, com velocidade de afastamento entre cargas de $100 \pm 10 \mathrm{~mm} / \mathrm{min}$. A célula de carga utilizada no dinamômetro foi de $200 \mathrm{kgf}$. A calibração foi realizada pela Emic-Dcame, laboratório de calibração credenciado pela

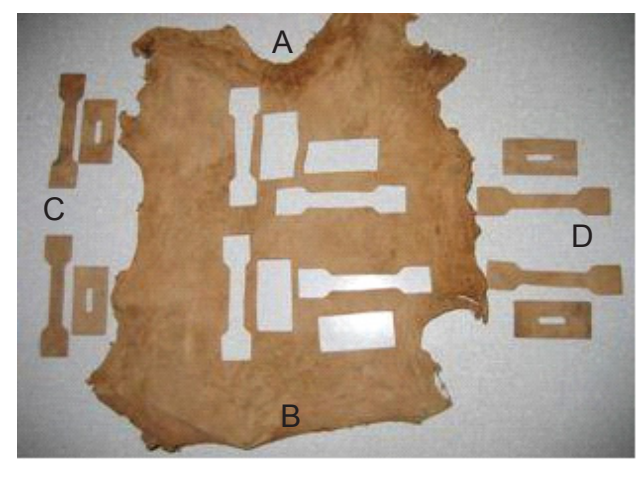

Figura 1. Posição utilizada para a retirada dos corpos de prova do couro de coelhos. A: Região anterior, $B$ : Região posterior; $C$ : Corpos de prova para tração e alongamento retirados na posição longitudinal; D: Corpos de prova para rasgo retirados na posição transversal. (Position used to taken samples of rabbit skins. A: Front region, B: Rear region, C: Samples for traction and stretching test, taken from longitudinal direction. D: Samples for tearing test taken from transversal direction).
Cgcre/Inmetro sob $\mathrm{n}^{\circ}$ 197. O número do certificado de calibração da célula de carga do dinamômetro é 288/08 emitida em 16 de maio de 2008.

Foi utilizado um delineamento inteiramente casualizado em esquema de parcelas subdivididas, tendo nas parcelas os tratamentos $(\mathrm{T}=$ tempo normal; $\mathrm{T} 2=$ tempo contínuo) e nas subparcelas as posições de corte no couro $(\mathrm{P} 1=$ anterior longitudinal; $\mathrm{P} 2=$ anterior transversal; $\mathrm{P} 3=$ posterior longitudinal e $\mathrm{P} 4=$ posterior transversal), com dez repetições por tratamento. Os resultados dos testes físico-mecânicos foram submetidos à análise de variância, sendo as médias comparadas pelo teste de Tukey utilizando-se o programa estatístico SAEG (2000) ao nível de 5\% de significância.

Para avaliação qualidade de resistência dos couros de coelhos curtidos neste experimento, os resultados estatísticos obtidos foram comparados com a recomendação da BASF (2005) para couros aplicados em vestuário. Pois, de acordo com BASF (2005), os valores referência para couros curtidos ao cromo para vestuário, independentemente do tipo de recurtimento, deve ser de, no mínimo de $25 \mathrm{~N} / \mathrm{mm}^{2}$ de resistência à tração ou tensão e no mínimo $35 \mathrm{~N} / \mathrm{mm}$ para a resistência de rasgamento progressivo. As exigências (provisórias) de qualidade para couro de vestimenta, estabelecidas pela Comissão de Especificação dos Institutos de Couro, para camurça, nubuk e couro napa acabado são, respectivamente, $15 \mathrm{~N} / \mathrm{mm}$ e $20 \mathrm{~N} / \mathrm{mm}$ para resistência ao rasgamento progressivo. Para a resistência à tração, o valor deve ser de $12 \mathrm{~N} / \mathrm{mm}^{2}$ para os dois tipos de produtos mencionados.

\section{RESULTADOSEDISCUSSÃO}

A tabela II apresenta a espessura dos couros de coelhos. Os couros de coelhos submetidos à técnica de curtimento com tempo contínuo apresentaram uma maior espessura $(p<0,05)$, pode-se inferir que os produtos químicos utilizados não chegaram 


\section{TEMPOS DAS ETAPAS NO CURTIMENTO}

reagir totalmente com as fibras colágenas, ficando camadas sobrepostas dos reagentes utilizados. O processo de curtimento com o tempo normal (com intervalos de descanso no curtimento e após basificação) proporcionou um maior tempo de reação dos produtos químicos com as fibras colágenas. Também houve diferença $(\mathrm{p}>0,05)$ para a espessura nas diferentes posições do couro, pois quando retirado os corpos de provas nas diferentes posições avaliadas, pode-se observar que houve diferença estatística (tabela II). A espessura do couro na região anterior na posição longitudinal foi menor enquanto a maior espessura foi na região posterior na posição transversal, apesar de não ter diferido significativamente da região anterior transversal e posterior longitudinal. Isto demonstra que o couro não se apresentou homogêneo em toda sua extensão independente do tempo utilizado no processo de curtimento. Portanto, ocorre uma variação na espessura do couro ao longo de seu revestimento sobre o animal.

Oliveira et al. (2007) relataram que a espessura dos couros dos coelhos com 70 dias foram 1,02 mm e 1,15 mm, enquanto para os animais com 400 dias de 2,21 $\mathrm{mm}$ e 1,69 $\mathrm{mm}$, respectivamente para machos e fêmeas. No experimento de Oliveira et al. (2007) foi utilizado o tempo normal de curtimento, em termos dos tempos nas etapas do processo e repouso entre etapas. Neste experimento a média de espessura dos couros foi de $0,97 \mathrm{~mm}$ independente da posição de retirada dos corpos de prova ou tempo de curtimento, valor este, próximo a espessura dos couros dos animais de 70 dias relatados por Oliveira et al. (2007).

Houve interação para força máxima aplicada nos testes, tração e alongamento e rasgamento progressivo, e tais resultados estão na figura 2. Avaliando as regiões do couro independente do tempo de processamento, a região posterior na posição longitudinal apresentou menor resistência (tempo normal $=6,01 \mathrm{~N} / \mathrm{mm}$ e tempo contínuo $=3,54 \mathrm{~N} / \mathrm{mm}$ ). No tempo contínuo
Tabela II. Médias e desvios-padrão para espessura (mm), considerando o tempo de curtimento e as posições de retirada dos corpos de prova no couro. (Means and standard deviation for thickness, considering tanning time and position of samples in skin).

\begin{tabular}{lc}
\hline Região e posição & \\
Anterior longitudinal & $0,88 \pm 0,11^{\mathrm{B}}$ \\
Anterior transversal & $0,96 \pm 0,15^{\mathrm{AB}}$ \\
Posterior longitudinal & $1,02 \pm 0,13^{\mathrm{AB}}$ \\
Posterior transversal & $1,04 \pm 0,20^{\mathrm{A}}$ \\
Média geral & $0,97 \pm 0,16$ \\
Tempo de curtimento & \\
Normal & $0,89 \pm 0,11^{\mathrm{A}}$ \\
Continuo & $1,063 \pm 0,15^{\mathrm{B}}$ \\
C.V. $(\%)$ & 11,67 \\
\hline
\end{tabular}

${ }^{A B}$ Médias seguidas de mesmas letras não diferem entre si pelo teste de Tukey $(p>0,05)$.

às regiões anterior e posterior transversal apresentaram maior resistência a tração (figura 2), enquanto no tempo normal não houve diferença entre a região anterior longitudinal e transversal e a região posterior transversal. A média do teste de determinação da tração para o tempo de curtimento normal foi $8,81 \mathrm{~N} / \mathrm{mm}^{2}$ e o contínuo $7,69 \mathrm{~N} / \mathrm{mm}^{2}$. Portanto, o curtimento em tempo normal proporcionou uma resistência de $12,72 \%$ a mais quando comparado ao tempo contínuo. Podemos inferir que no tempo normal devido ao maior período de realização do processo de curtimento e os tempos de repouso entre determinadas etapas, proporcionaram maiores condições dos produtos químicos reagirem com as fibras colágenas, associado à característica intrínseco da estrutura da pele, ou seja, a pele na região anterior é mais resistente que a posterior e a posição mais frágil é a longitudinal. Estando isso diretamente relacionado com a orientação ou distribuição das fibras, bem como até a espessura e quantidades de fibras nesta região.

De acordo com Oliveira et al. (2007) os couros de coelhos não apresentaram 


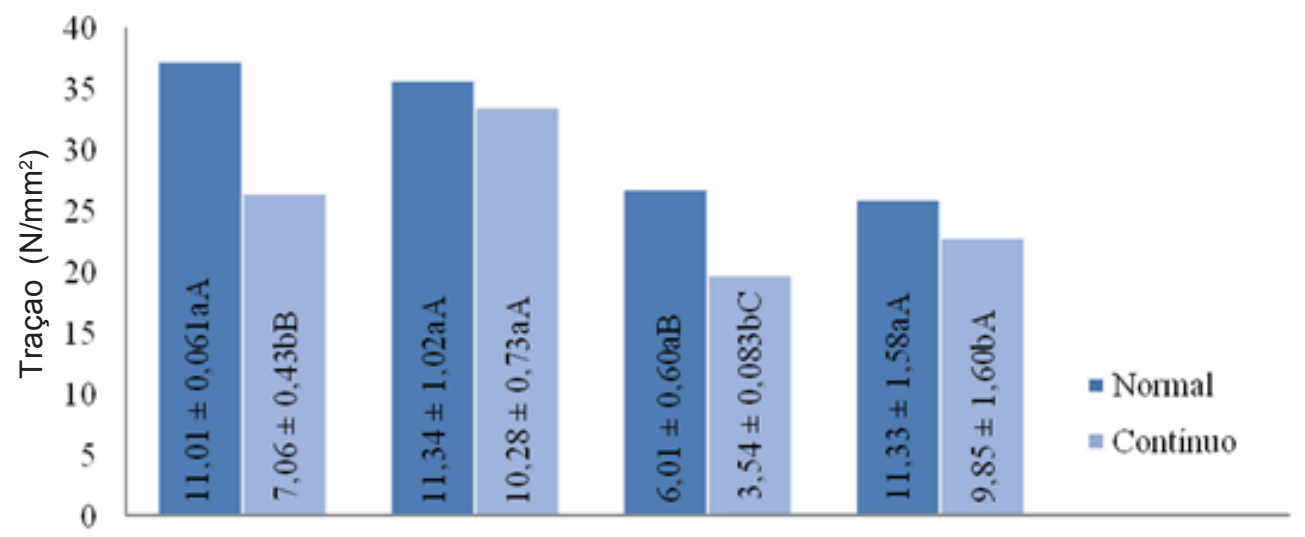

A. Longitudinal A. Transversal P. Longitudinal P. Transversal

Figura 2. Desdobramento da interação para a determinação da tração $\left(\mathrm{N} / \mathrm{mm}^{2}\right)$. Letras minúsculas iguais para cada tempo de curtimento dentro de cada posição de retirada do corpo de prova, não diferem entre si pelo teste de Tukey $(p>0,05)$. Letras maiúsculas para cada posição dentro de cada tempo de curtimento, não diferem entre si pelo teste de Tukey $(p>0,05)$. Coeficiente de variação 9,9\%. (Unfolding of the interaction for determine the tensile strength $\left(\mathrm{N} / \mathrm{mm}^{2}\right)$. Small letters for each tanning time within each position of the samples, do not differ by Tukey test $(p>0.05)$. Capital letters for each position within each time tanning, do not differ by Tukey test $(p>0.05)$. Coefficient of variation $9.9 \%)$.

diferença significativa para a posição de retirada do corpo de prova (longitudinal= $7,25 \mathrm{~N} / \mathrm{mm}^{2}$ e transversal $=8,04 \mathrm{~N} / \mathrm{mm}^{2}$ ). Os autores utilizaram a mesma metodologia aplicada no curtimento normal deste experimento, com apenas aumento de $1 \%$ de sulfeto de sódio. Eles trabalharam com peles de coelhos de 70 dias e 400 dias, machos e fêmas. Os resultados obtidos neste experimento se aproximaram dos relatados por Oliveira et al. (2007), apesar das variáveis que eles utilizaram (idade e sexo).

Hoch et al. (2009) estudaram a ação de diferentes agentes curtentes utilizados no curtimento de peles de coelhos abatidos com 70 dias de idade. Os autores relataram que a tração variou de 9,28 a 11,63 N/mm² entre tratamentos avaliados, enquanto os couros no sentido longitudinal apresentaram uma resistência de $10,96 \mathrm{~N} / \mathrm{mm}^{2}$ e o transversal 9,95 N/mm², para couros com espessura variando de 0,92 a 1,10 mm. Estes valores foram superiores aos obtidos neste experimento para a região posterior longitudinal, independente do tempo de curtimento (figura 3).

Jacinto et al. (2000), analisando os couros de novilhas do Pantanal Sul Mato Grossense, com espessura mínima no sentido longitudinal de $1,68 \mathrm{~mm}$ e $2,01 \mathrm{~mm}$ na transversal, obtiveram couro com resistência à tração de $20,13 \mathrm{~N} / \mathrm{mm}^{2}$ e $14,14 \mathrm{~N} / \mathrm{mm}^{2}$, respectivamente para esses dois sentidos. Já, os couros de coelhos analisados neste experimento apresentaram uma espessura variando de $0,88 \mathrm{~mm}$ a $1,04 \mathrm{~mm}$. Para essas espessuras a resistência a tração variou de $3,54 \mathrm{~N} / \mathrm{mm}^{2}$ a $11,34 \mathrm{~N} / \mathrm{mm}^{2}$. Segundo Jacinto et al. (2000), os couros de novilhas, são mais resistentes, a tração no sentido longitudinal. No couro de coelho, para essa característica, a posição longitudinal da região posterior apresentou menor resistência (figura 2). Contudo, pode-se observar que os 


\section{TEMPOS DASETAPASNO CURTIMENTO}

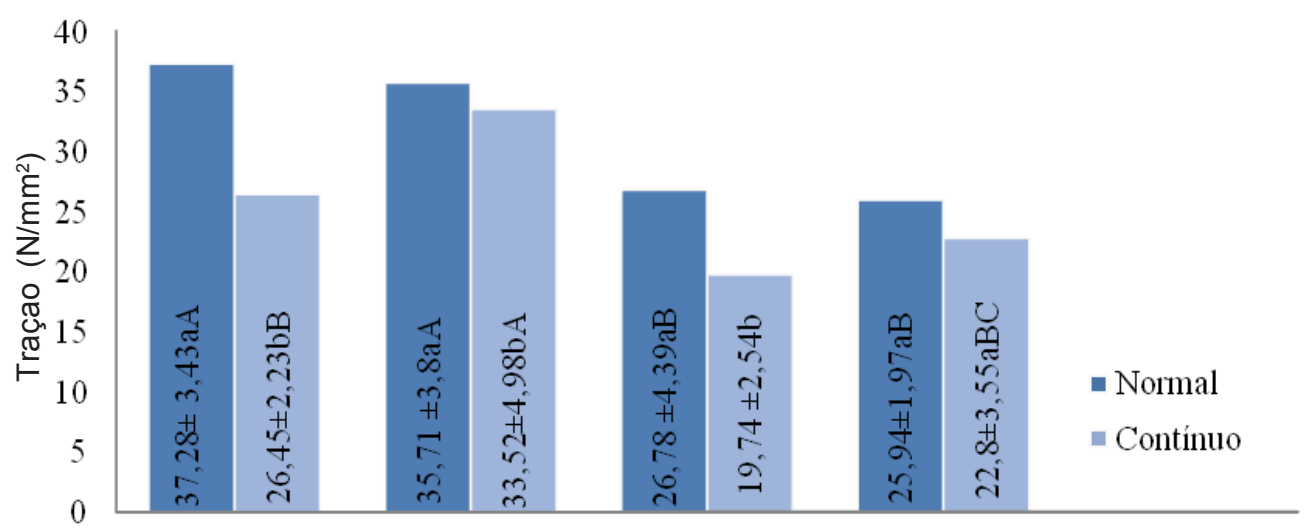
A. Longitudinal
A. Transversal
P. Longitudinal
P. Transversal

Figura 3. Desdobramento da interação para a determinação do rasgamento progressivo (N) $\mathrm{mm}$ ). Letras minúsculas iguais para cada tempo de curtimento dentro de cada posição de retirada do corpo de prova, não diferem entre si pelo teste de Tukey $(p>0,05)$. Letras maiúsculas para cada posição dentro de cada tempo de curtimento, não diferem entre si pelo teste de Tukey $(p>0,05)$. Coeficiente de variação $(10,77 \%)$. (Unfolding of the interaction for determine progressive tearing $(\mathrm{N} / \mathrm{mm})$. Small letters for each tanning time within each position of the samples, do not differ by Tukey test $(p>0.05)$. Capital letters for each position within each time tanning, do not differ by Tukey test ( $p>0.05)$. Coefficient of variation).

couros de coelhos, apesar de apresentarem em torno da metade do valor da espessura média dos couros de novilhas, os couros de coelhos apresentaram resistência a tração no sentido transversal (anterior $=11,34 \mathrm{~N} /$ $\mathrm{mm}^{2}$ e posterior $\left.=11,33 \mathrm{~N} / \mathrm{mm}^{2}\right)$ e longitudinal anterior $\left(11,01 \mathrm{~N} / \mathrm{mm}^{2}\right)$ muito semelhante aos de novilhas quando curtidos com tempo normal (figura 2).

Jacinto et al. (2005) relatam as características fisico-mecânicas do couro de ovinos através do processo de curtimento. De acordo com os autores, couros com espessura de 1,22 e 1,27 mm obtidos de animais das raças Morada Nova e Ideal, apresentaram resistência à tração de 15,35 e $12,68 \mathrm{~N} / \mathrm{mm}^{2}$, respectivamente para a posição longitudinal e transversal. O valor obtido para a posição transversal de ovinos se assemelha aos encontrados, neste experimento, para os couros de coelhos na mesma posição, enquanto na posição longitudinal, os couros de ovelhas apresentaram uma maior resistência comparada ao do couro de coelho. Portanto, os couros ovinos são mais resistentes na posição longitudinal, necessitando de $187,10 \mathrm{~N}$ de carga de força para o teste de tração à ruptura (Jacinto et al., 2005). Para os couros de coelhos, neste experimento, o valor variou de 34,20 a 117,80 N(tabela III).

Para o rasgamento progressivo, o tempo de curtimento normal proporcionou uma resistência aos couros de $18,43 \%$ superior comparado aos curtidos no tempo contínuo. As médias de rasgamento obtidas entre os tempos de curtimento foram $31,43 \pm 6,15 \mathrm{~N} /$ $\mathrm{mm}$ e $25,63 \pm 6,17 \mathrm{~N} / \mathrm{mm}$, respectivamente para normal e contínuo. Levando-se em consideração o tempo de curtimento, apenas na região posterior transversal (nor$\mathrm{mal}=25,94 \mathrm{~N} / \mathrm{mm}$; contínuo $=22,80 \mathrm{~N} / \mathrm{mm}$ ) o couro não apresentou diferença significativa para rasgamento progressivo (figura 3 ). 
Tabela III. Médias e desvios-padrão da força máxima, determinação da tração, alongamento e rasgamento progressivo, considerando o tempo de curtimento e as posições dos corpos de prova no couro. (Means and standard deviation of maximum force, traction, elongation and progressive tearing determination, considering the tanning time and positions of samples in the leather).

\begin{tabular}{|c|c|c|}
\hline \multirow[t]{2}{*}{ Características } & \multicolumn{2}{|c|}{ Tempo de curtimento } \\
\hline & Normal & Contínuo \\
\hline Força máxima $(\mathrm{N})$ & & \\
\hline Anterior longitudinal & $73,80 \pm 8,14^{\mathrm{aB}}$ & $62,20 \pm 6,42^{\mathrm{aB}}$ \\
\hline Anterior transversal & $103,20 \pm 14,84^{\mathrm{aA}}$ & $108,80 \pm 12,46^{\mathrm{a} A}$ \\
\hline Posterior longitudinal & $48,80 \pm 6,68^{a c}$ & $34,20 \pm 2,50^{\mathrm{bc}}$ \\
\hline Posterior transversal & $96,80 \pm 2,95^{\mathrm{bA}}$ & $117,80 \pm 6,30^{\mathrm{aA}}$ \\
\hline Média & $80,65,18 \pm 23,54$ & $80,75 \pm 35,78$ \\
\hline Média geral & \multicolumn{2}{|c|}{$80,7 \pm 29,89$} \\
\hline C.V. & \multicolumn{2}{|c|}{$11,4 \%$} \\
\hline \multicolumn{3}{|l|}{ Alongamento (\%) } \\
\hline Anterior longitudinal & $61,00 \pm 7,87^{\mathrm{bBC}}$ & $78,20 \pm 12,74^{\mathrm{aB}}$ \\
\hline Anterior transversal & $55,00 \pm 4,85^{\mathrm{bC}}$ & $86,40 \pm 6,90^{\mathrm{aAB}}$ \\
\hline Posterior longitudinal & $67,80 \pm 5,50^{\mathrm{aAB}}$ & $64,60 \pm 6,88^{\mathrm{ac}}$ \\
\hline Posterior transversal & $77,00 \pm 2,74^{\mathrm{bA}}$ & $96,80 \pm 3,35^{\mathrm{aA}}$ \\
\hline Média & $65,20 \pm 9,82$ & $81,50 \pm 14,53$ \\
\hline Média geral & \multirow{2}{*}{\multicolumn{2}{|c|}{$\begin{array}{c}73,35 \pm 14,62 \\
9,88 \%\end{array}$}} \\
\hline C.V. & & \\
\hline
\end{tabular}

abABCMédias seguidas de mesmas letras minúsculas nas linhas e maiúsculas nas colunas não diferem entre si pelo teste de Tukey $(p>0,05)$.

Portanto, nas regiões anterior longitudinal e transversal e na região posterior longitudinal foram significativamente mais resistentes no tempo normal quando comparado ao tempo contínuo. Quando comparado as regiões dentro de cada tempo de curtimento, observa-se na figura 3 , para o curtimento normal, que a região anterior apresentou mais resistência que a região posterior do couro, independente do sentido de retirada do corpo de prova. No curtimento contínuo a região posterior também apresentou a menor resistência para rasgamento, porém, a maior resistência foi na região anterior transversal (figura 3).

De acordo com Oliveira et al. (2007), os couros de coelhos abatidos com 70 dias de idade, machos e fêmeas, apresentaram uma resistência ao rasgamento progressivo de 14,06 e $13,46 \mathrm{~N} / \mathrm{mm}$, respectivamente. Hoch et al. (2009), relatam que os couros de coelhos apresentaram uma resistência ao rasgamento progressivo variando de 10,34 $\mathrm{N} / \mathrm{mm}$ a $25,94 \mathrm{~N} / \mathrm{mm}$ entre os tratamentos onde foi avaliada a ação dos diferentes curtentes no couro. Valores estes muito inferiores aos obtidos neste experimento (figura 3).

Os valores de resistência ao rasgamento progressivo (longitudinal $=62,43 \mathrm{~N} / \mathrm{mm}$ e transversal $=68,36 \mathrm{~N} / \mathrm{mm})$, citado por Jacinto et al. (2000), para couros de novilhas do Pantanal Sul Mato Grossense, são muito superiores aos obtidos neste experimento para couros de coelhos. Também, Jacinto et al. (2005), relatam que couros de ovinos Morada Nova e Ideal, apresentaram maior resistência para o rasgamento progressivo na posição transversal $(60,87 \mathrm{~N} / \mathrm{mm})$. Para os couros de coelhos os valores foram muito inferiores (tempo contínuo $=19,74 \mathrm{~N} / \mathrm{mm}$ a tempo normal $=37,28 \mathrm{~N} / \mathrm{mm}$ ), quando com- 


\section{TEMPOS DAS ETAPAS NO CURTIMENTO}

parados com os resultados dos couros de novilhas (Jacinto et al., 2000) e ovinos Morada Nova e Ideal (Jacinto et al., 2005).

Os valores obtidos para alongamento, mostraram que o couro de coelho apresentou elevada elasticidade (tabela III). Apesar da menor elasticidade observada com a aplicação do curtimento em período normal, os valores se encontram dentro dos padrões exigidos para sua utilização na confecção de vestuários quando comparados com o relato da BASF (2005), onde no teste de alongamento é recomendado no mínimo $60 \%$ até a ruptura do couro.

Os couros curtidos no tempo normal apresentaram 20\% menos elasticidade quando comparado ao tempo contínuo de curtimento. Quando analisado o tempo de curtimento dentro das regiões e posições de retirada do corpo de prova, as regiões anterior longitudinal e transversal e região posterior transversal apresentaram maior elasticidade no tempo contínuo, enquanto para região posterior longitudinal não houve diferença significativa entre os tempos de processo.

Quando analisado as regiões e posições do couro dentro de cada tempo de processamento de curtimento, pode-se observar na tabela III, que a região anterior transversal apresentou menor elasticidade não diferindo da região anterior longitudinal. Todavia, a maior elasticidade foi para a região posterior transversal não diferindo da região posterior longitudinal, quando os couros foram curtidos no tempo normal. Para o curtimento contínuo, a maior elasticidade foi na região posterior transversal e a menor na posterior longitudinal.

Os couros de coelhos machos e fêmeas, abatidos com 70 dias segundo Oliveira et al. (2007), apresentaram uma elasticidade de $53 \%$ e $50,25 \%$, respectivamente, sendo estes resultados muito inferiores aos obtidos neste experimento, principalmente quando comparado ao curtimento de tempo contínuo (tabela III). Todavia, quando avaliado a ação de diferentes curtentes para couros de coelhos, a elasticidade do couro variou de $37,83 \%$ a 70,28\% (Hoch et al., 2009). Sendo que a elasticidade dos couros neste experimento foram superiores comparado ao menor valor relatado por Hoch et al. (2009).

De acordo com Jacinto et al. (2000), os valores máximos de alongamento obtidos para novilhas foram de $64 \%$ e $82 \%$, respectivamente para sentido longitudinal e transversal. Os couros de coelhos apresentaram um valor médio de alongamento de $55 \%$ a $96,8 \%$. Todavia, a elasticidade do couro de coelho no sentido transversal independente de ser região anterior ou posterior, apresentou maior elasticidade quando os couros foram curtidos com tempo contínuo, assemelhando-se ao valor obtido para novilha.

A força máxima aplicada no teste de tração e alongamento diferiu ( $p>0,05)$ entre os tratamentos para a região posterior longitudinal e transversal, sendo a elasticidade maior $(48,80 \%)$ e menor $(96,89 \%)$, respectivamente para as duas posições, no tempo normal de curtimento.

Quando analisada a força máxima aplicada em cada posição do couro dentro de cada tempo de curtimento, a região anterior transversal necessitou de maior força $(p<0,05)$ para a ruptura do couro ao teste de tração e alongamento $(103,20 \mathrm{~N})$, apesar de não ter diferido da posição posterior transversal $(\mathrm{P} 1=96,80 \mathrm{~N})$, no tempo normal. No tempo contínuo a região anterior e posterior transversal apresentaram maior elasticidade comparado as demais.

De acordo com Oliveira et al. (2007) os couros de coelhos apresentaram uma força de $84,55 \mathrm{~N}$ para sentido longitudinal e 128,41 $\mathrm{N}$ para transversal, ou seja, o couro no sentido transversal necessitou de maior força até a ruptura do couro, para realizar os testes de determinação de tração e alongamento. O mesmo ocorreu neste experimento, onde os couros no sentido transversal apresentaram maiores valores de força máxima, independente do tempo de curtimento. 
Essas variações encontradas para as determinações de resistência dos couros, podem estar diretamente relacionadas com a distribuição, orientação e disposição das fibras colágenas, uma vez que a espessura dos couros de ovinos apresentaram valores muito próximos aos de coelhos analisados.

Comparando os resultados obtidos neste trabalho com os valores de referência para confecção de vestuário, de acordo com BASF (2005), o couro de coelho não poderia ser utilizado para tal finalidade. No entanto, a resistência dos couros de coelho foram superiores aos valores estabelecidos pela Comissão de Especificação dos Institutos de Couro, para camurça, nubuk e couro napa acabado para aplicação em vestimentas quanto à resistência ao rasgamento progressivo.

O processo de curtimento contínuo, conduzindo as etapas de processamento sem tempo de repouso (entre as etapas) e com algumas alterações no tempo do processo (tempo mínimo a ser realizado na etapa do processo), permite o menor custo de produção. Em função das possibilidades de otimização no uso de equipamentos de curtumes, torna-se interessante fazer novas avaliações no processo de curtimento das peles de coelho, alterando a formulação, em relação as quantidades ou tipos de produtos químicos utilizados, ou tempo de execução de cada etapa buscando reduzir, ao máximo, o tempo total de processamento de cada lote de peles.

\section{CONCLUSÃO}

Considerando a formulação utilizada neste experimento, os couros devem ser curtidos pelo método com tempo normal de processamento, por proporcionarem couros com resistência a tração e rasgamento superiores aos curtidos em tempo contínuo de processamento. A região anterior apresentou maior resistência comparada a região posterior para rasgamento progressivo, independente do tempo de curtimento utilizado. Os couros na região posterior longitudinal apresentaram menor resistência a tração, independente do tempo de processamento aplicado. O curtimento em período normal proporcionou couros com menor elasticidade, mas pelo teste de rasgamento progressivo e alongamento, os valores estão dentro dos padrões exigidos para confecção de vestuários seguindo os valores BASF (2005).

\section{AGRADECIMENTOS}

Á Secretaria Especial de Aqüicultura e Pesca da Presidência da República, atual Ministério da Pesca e Aquicultura, pelo apoio na obtenção dos equipamentos do laboratório de curtimento da Universidade Estadual de Maringá, possibilitando a realização desse experimento.

\section{BIBLIOGRAFIA}

ABNT. 1997. Associação Brasileira de Normas Técnicas. NBR 11041: couros - determinação da resistência à tração e alongamento. Rio de Janeiro.

ABNT. 2005a. Associação Brasileira de Normas Técnicas. NBR 11055: couro - determinação da força de rasgamento progressivo. Rio de Janeiro.

ABNT. 2005b. Associação Brasileira de Normas Técnicas. NBR 11052: couro determinação da espessura. Rio de Janeiro.

ABNT. 2006. Associação Brasileira de Normas

Técnicas. NBR 10455: climatização de materiais usados na fabricação de calçados e correlatos. Rio de Janeiro.

BASF. 2005. Vademécum do curtidor. $4^{\mathrm{a}}$ ed. Rev. Amp. Ludwighafen. 455 pp.

Carabaño, R. 2000. Sistemas de producción de conejos en condiciones intensivas. Reunião Anual da Sociedade Brasileira de Zootecnia SBZ. Viçosa. Brasil. 37: 17-37.

Fernandéz-Carmona, J., Pascual, J.J. and Cervera, C. 2000. The use of fat in rabbit diets. World Congress of Animal Feeding. ACAF. Valencia.

Archivos de zootecnia vol. 61, núm. 235, p. 434. 


\section{TEMPOS DAS ETAPAS NO CURTIMENTO}

España. 7: 29-59.

Hoch, A.L.V., Prado, M., Franco, M.L.R.S., Scapinello, C., Franco, N.P. e Gasparino, E. 2009. Ação de diferentes agentes curtentes utilizados no curtimento de peles de coelhos: testes físico-mecânicos dos couros. Acta Sci, 31: 411-415.

Hoinacki, E. 1989. Peles e couros - origens, defeitos e industrialização. Henrique d'Ávila Bertaso, $2^{\text {a }}$ ed. Porto Alegre.

Jacinto, M.A.C., Fornari, C.J.S., Farias, F.J.C., Torres, R.D. e Medeiros, E.M.C. 2000. Aspectos qualitativos do couro de novilha orgânica do Pantanal Sul-Mato-Grossense. Circular técnica 33. http://www.cnpgc.embrapa.br/publicacoes/ ct/ct33/index.htm (22/02/2010).

Jacinto, M.A.C., Costa, R.G. e Leite, E.R. 2005. Produção de peles e couros caprinos e ovinos.
Reunião Anual da Sociedade Brasileira de Zootecnia, 42. Anais... Sociedade Brasileira de Zootecnia. Goiânia. pp. 157-165.

Oliveira, A.C., Souza, M.L.R., Hoch, A.L.V., Gasparino, E., Scapinello, C., Kffuri, V.R. e Domingues, M.C. 2007.Resistência dos couros de coelhos em função do sexo e da idade de abate. Tecnicouro, 28: 52-56.

SAEG. 2000. Sistemas de Análises Estatísticas e Genéticas. Versão 8,0. Universidade Federal de Viçosa. Viçosa.

Scapinello, C. 1986. Atualização em Cunicultura. Cooperativa Norte Paranaense de Criadores de Coelho Ltda. Coopernorte Coelhos. Maringá, PR.

Souza, M.L.R. 2004. Tecnologia para processamento das peles de peixe. Maringá, PR. Coleção Fundamentum, 11: 14-55. 